\title{
O USO DE AZEITE DE DENDÊ NA FORTIFICAÇÃO DE ALIMENTOS ${ }^{1}$
}

\author{
Patrícia Helena Cravo Rodrigues², Maria Elisabeth Machado Pinto-e-Silva ${ }^{3}$, \\ Sophia Cornbluth Szarfarc ${ }^{3}$
}

O objetivo do trabalho foi incluir o azeite de dendê como fonte de vitamina A em alimentos de consumo habitual da população brasileira e avaliar sua estabilidade e aceitação. Foram desenvolvidas as preparações: sequilho salgado, sequilho doce e biscoito de polvilho em suas formulações originais e as acrescidas de azeite de dendê. A vitamina A foi determinada pela quantificação de $\beta$ caroteno presente nas amostras do azeite de dendê e nas preparações. Para a análise sensorial, utilizou-se o teste afetivo de aceitabilidade. As preparações desenvolvidas tiveram como parâmetro as originais para posterior análise. Com relação ao teor de vitamina $\mathrm{A}$, todos os produtos com dendê tiveram concentrações significativas deste nutriente quando comparados aos biscoitos sem dendê. Pela análise sensorial, os produtos apresentaram aceitabilidade no atributo aceitação global. O azeite de dendê se manteve como fonte de vitamina A quando utilizado como ingrediente de preparações, mesmo se submetido a um processamento térmico, e mostrou ser uma boa fonte complementar deste nutriente. Assim, o azeite de dendê pode ser utilizado no enriquecimento de alimentos pela sua contribuição em retinol visando o controle da hipovitaminose A.

Palavras-chave: vitamina A, hipovitaminose A, azeite de dendê, fortificação.

\section{USE OF PALM OIL IN FOOD FORTIFICATION}

The objective of the study was to include the palm oil as vitamin A source to make general consumption food in Brazil and to assess the food acceptation. The following preparations were developed: salt starchy biscuit, sweet starchy biscuit and cookies made from cassava flour. The vitamin A content was determined by $\beta$ carotene quantification in samples. All the developed preparations were submitted to a sensorial analysis. To sensorial analysis, it was used the emotional test of acceptance. All developed preparations were compared with their original products. All products with palm oil have shown significant vitamin A concentrations when compared with biscuits with no palm oil. According to sensorial analysis, all products had good acceptance. The palm oil continued as a vitamin A source when used as a food preparation ingredient, even when submitted to thermal processing, showing to be an excellent additional source of this nutrient. Therefore, palm oil is a powerful vitamin A source and can be used in food fortification aimed at vitamin A deficiency control.

Key-words: vitamin A, vitamin A deficiency, palm oil, fortification.

\footnotetext{
${ }^{1}$ Auxílio financeiro do Conselho Nacional de Desenvolvimento Científico e Tecnológico (CNPq). Verba de bancada da bolsa de produtividade de Szarfarc, SC (Processo № 303249/87).

${ }^{2}$ Departamento de Nutrição. Faculdade de Saúde Pública, Universidade de São Paulo (USP). Cerqueira César, São Paulo, SP, Brasil. Correspondência: Rua Dr. Virgílio de Carvalho Pinto 381/104, Pinheiros, CEP 05415-030, São Paulo, SP. E-mail: patriciacravo@usp.br

${ }_{3}^{3}$ Departamento de Nutrição. Faculdade de Saúde Pública, USP.
} 


\section{INTRODUÇÃO}

A hipovitaminose A é reconhecida mundialmente como um dos problemas de saúde pública da maior relevância pela elevada frequência com que ocorre e consequências deletérias que acarreta especialmente entre crianças em idade pré-escolar. A Deficiência de Vitamina A (DVA) é reconhecida como a principal causa de cegueira noturna evitável [1] e mesmo no caso de deficiência leve, pode haver comprometimento do sistema imunológico, o que reduz a resistência à diarreia e ao sarampo ${ }^{[2]}$. Segundo Sarni et al. ${ }^{[3]}$, a deficiência de vitamina A está associada a $23 \%$ de mortalidade por diarreia no mundo.

De acordo com Geraldo et al. ${ }^{[4]}$, estima-se que mais de 250 milhões de crianças em todo mundo apresentem baixas reservas de vitamina A.

No Brasil, são poucos os estudos referentes à hipovitaminose $\mathrm{A}$ e, normalmente, centralizados nas regiões Nordeste e Sudeste. A diversidade de indicadores utilizados nesses estudos, juntamente com a limitação geográfica das informações não permitem que o problema possa ser dimensionado em sua extensão e/ou magnitude.

Em 2009, a Pesquisa Nacional de Demografia e Saúde da Criança e da Mulher (PNDS) ${ }^{[5]}$ apresentou um retrato da prevalência da hipovitaminose $\mathrm{A}$ em crianças de 6 a 59 meses de idade e em mulheres em idade fértil. O estudo, fundamentado no uso de indicadores apropriados e devidamente padronizados, permitiu uma visão da extensão e magnitude do problema. Destacam os autores do levantamento que, embora não sendo possível analisar a tendência da situação de hipovitaminose A por não haver outra pesquisa nacional, os dados obtidos servirão de referência para a avaliação de intervenções indispensáveis para atender o compromisso firmado pelo Brasil junto às Nações Unidas em 1992.

Além de apresentar a grande prevalência de DVA entre a população infantil de todas as regiões brasileiras, os dados da PNDS ressaltam que esta deficiência não é exclusiva em crianças de baixa idade, mas ocorre entre mulheres em idade reprodutiva o que, por sua vez, permite supor que toda a família da qual a mulher faz parte seja vulnerável à hipovitaminose $\mathrm{A}$ $\mathrm{e}$ às suas consequências ${ }^{[5]}$.
O principal determinante da DVA, segundo estudos epidemiológicos, é a inadequação do consumo alimentar ${ }^{[6]}$. Dentro de um modelo de evolução da desnutrição, o consumo alimentar insuficiente corresponde à primeira fase da deficiência e, assim sendo, o atendimento da necessidade nutricional da vitamina constitui uma intervenção precoce que evitará que os sinais bioquímicos e clínicos da patologia se instalem ${ }^{[7]}$.

Em um levantamento dos estudos relacionados à DVA no Brasil, utilizando os métodos clínicos, bioquímicos e dietéticos, desde a década de 60, Geraldo et al. ${ }^{[4]}$ ressaltaram que, independente do critério utilizado para o diagnóstico da deficiência, verificou-se uma associação entre a hipovitaminose A e a ocorrência de morbidades e mortalidade, principalmente entre crianças em idade pré-escolar.

Diante do exposto, fica evidente a necessidade da busca de alternativas que possam atender à família como um todo no controle da hipovitaminose A. A escolha do azeite de dendê como veículo da vitamina A levou em conta ser este fonte relevante do nutriente, apto a suprir, mesmo quando utilizado em quantidades mínimas, fração significativa da necessidade nutricional. Entre os óleos vegetais mundialmente consumidos, o azeite de dendê é aquele que contém a maior concentração conhecida de carotenoides com atividade de vitamina $\mathrm{A}$, sendo que o principal deles, o $\beta$ caroteno, constitui $60 \%$ do total dos pigmentos ${ }^{[8]}$. O $\beta$ caroteno possui uma maior capacidade de conversão para a vitamina $A$, estando em torno de $50 \%{ }^{[8-10]}$.

Assim sendo, este estudo teve como objetivo verificar se a inclusão do azeite de dendê em preparações habituais, através da substituição parcial de óleos/gorduras por este, contribuiria no atendimento das necessidades nutricionais da vitamina A e, dessa forma, propiciaria uma melhor qualidade de vida para a população brasileira.

\section{METODOLOGIA}

O projeto do estudo foi submetido e aprovado pelo Comitê de Ética em Pesquisa da Faculdade de Saúde Pública da Universidade de São Paulo, conforme as normas da Resolução 196, de 10/10/1996, do Conselho Nacional de Saúde, que regulamenta as pesquisas envolvendo seres humanos. 
Foram selecionadas para a adição de dendê as seguintes preparações: biscoito de polvilho, sequilho salgado e sequilho doce. A porcentagem de substituição da gordura pelo dendê teve como parâmetro de controle a intensidade da alteração no sabor comparado ao produto original, identificado por testes preliminares. A concentração de óleo/gordura e dendê em cada preparação está apresentada no Quadro. O modo de preparo foi similar para a receita original e a modificada (com adição de dendê).

\begin{tabular}{|lcccccc|}
\hline \multirow{2}{*}{ Ingrediente } & \multicolumn{6}{c|}{ Porcentagem de óleo/gordura e azeite de dendê } \\
\cline { 2 - 7 } & $\begin{array}{c}\text { Sequilho } \\
\text { Salgado }\end{array}$ & $\begin{array}{c}\text { Sequilho } \\
\text { Salgado com } \\
\text { dendê }\end{array}$ & $\begin{array}{c}\text { Sequilho } \\
\text { Doce }\end{array}$ & $\begin{array}{c}\text { Sequilho } \\
\text { Doce com } \\
\text { dendê }\end{array}$ & $\begin{array}{c}\text { Biscoito de } \\
\text { Polvilho }\end{array}$ & $\begin{array}{c}\text { Biscoito de } \\
\text { Polvilho com } \\
\text { dendê }\end{array}$ \\
\hline $\begin{array}{l}\text { Margarina } \\
\text { Óleo de milho }\end{array}$ & 18,4 & 4,6 & 13,3 & 3,2 & - & - \\
Azeite de dendê & - & - & - & - & 17,2 & 8,5 \\
\hline
\end{tabular}

Quadro. Porcentagem de óleo/gordura e de azeite de dendê utilizada nas formulações original e modificada.

O teor de vitamina A foi determinado a partir da concentração de $\beta$ caroteno presente nas amostras, segundo metodologia de Carvalho et al. [11]: o pigmento foi extraído da amostra de óleo/ gordura com acetona, saponificado com hidróxido de potássio metanólico, extraído com éter de petróleo e injetado no HPLC. As determinações foram feitas em triplicata.

Para que pudessem ser realizadas comparações entre as quantidades reais de vitamina A nos biscoitos crus e após o tratamento térmico, as porcentagens de retenção real de vitamina A foram calculadas utilizando a equação:

$$
\% \mathrm{RR}=\frac{\mathrm{Cp} \times 100}{\mathrm{Cc}}
$$

em que \% RR é a porcentagem de retenção real; Cp, conteúdo de vitamina A (mcg) por 100 gramas do alimento preparado, e Cc, conteúdo do nutriente $(\mathrm{mcg})$ por 100 gramas do alimento cru.

A análise sensorial foi determinada pela aplicação do teste afetivo de aceitabilidade, com escala hedônica de sete pontos. Os testes foram realizados com voluntários não treinados e o atributo sensorial foi avaliado sob condição controlada: em cabines individuais, amostras porcionadas em pratos descartáveis, acompanhadas de água potável para o enxague bucal entre as degustações. A ordem de apresentação das amostras foi aleatória e devidamente codificada. O produto foi considerado aceito quando alcançou uma porcentagem maior ou igual a $50 \%$, com valores variando entre "gostei moderadamente" a "gostei extremamente" da escala hedônica $(\geq 0,5)$.

Os resultados obtidos na análise sensorial foram avaliados por meio da análise de variância (ANOVA) [12].

\section{RESULTADOS}

Para a obtenção dos sequilhos e dos biscoitos com e sem dendê, adotou-se o mesmo processo produtivo, sendo percebidas apenas algumas mudanças nos produtos com dendê, dentre as quais estão a alteração na cor e na textura. Com relação à cor, a alteração ocorrida no produto com dendê já era esperada, em função da cor laranja-avermelhada que os carotenoides conferem ao azeite. Quanto à textura, a substituição parcial da gordura/óleo por azeite de dendê alterou a consistência da massa e a textura do produto final, ambos mais firmes. Possivelmente isto tenha ocorrido devido ao aumento do teor de ácidos graxos saturados presentes naturalmente no óleo de dendê, sendo semi-sólidos à temperatura ambiente, podendo, assim, contribuir com a firmeza observada.

A Tabela 1 apresenta os resultados da concentração de vitamina A nos produtos modificados, antes e após a cocção. Para os produtos "originais" verificou-se, como esperado, ausência de vitamina A. 
Tabela 1. Concentração de vitamina A, em retinol, segundo formulação modificada e do azeite de dendê utilizado no preparo dos alimentos, bem como a porcentagem de retenção.

\begin{tabular}{lccc}
\hline \multicolumn{1}{c}{ Produto } & $\begin{array}{c}\text { [vitamina A] } \mathbf{m c g} / \mathbf{1 0 0 g} \text { do } \\
\text { produto cru }\end{array}$ & $\begin{array}{c}\text { [vitamina A] } \mathbf{m c g} / \mathbf{1 0 0 g} \text { do } \\
\text { produto assado }\end{array}$ & $\begin{array}{c}\text { \% de retenção } \\
\text { de retinol }\end{array}$ \\
\hline Azeite de dendê & 2900 & - & - \\
Sequilho Salgado & 352,1 & 312,7 & 88,8 \\
Sequilho Doce & 248,1 & 230,7 & 93,0 \\
Biscoito de Polvilho & 403,9 & 341,3 & 84,5 \\
\hline
\end{tabular}

Com relação ao atendimento da necessidade de vitamina A, a Tabela 2 mostra que os produtos fornecem uma quantidade significativa deste nutriente.

Tabela 2. Percentual da Ingestão Diária Recomendada (IDR) ${ }^{[13]}$ em relação à vitamina $\mathrm{A}$, em $100 \mathrm{~g}$ e $30 \mathrm{~g}$ de Sequilho Salgado, Sequilho Doce e Biscoito de Polvilho com dendê.

\begin{tabular}{lcc}
\hline \multicolumn{1}{c}{$\begin{array}{c}\text { Amostra } \\
\text { (com dendê) }\end{array}$} & \multicolumn{2}{c}{ \% IDR* } \\
\cline { 2 - 3 } & $\mathbf{1 0 0 g}$ & $\begin{array}{c}\text { Porção de } \\
\mathbf{3 0 g}\end{array}$ \\
\hline Sequilho Salgado & 52,1 & 15,6 \\
Sequilho Doce & 38,4 & 11,5 \\
Biscoito de Polvilho & 56,9 & 17,1 \\
\hline
\end{tabular}

* IDR = Ingestão Diária Recomendada

A avaliação do grau de aceitação dos produtos através da análise sensorial por provadores não treinados, que analisaram os atributos segundo a escala hedônica de 7 pontos, estabeleceu valores atribuídos entre "gostei moderadamente" (valor 5) e "gostei extremamente" (valor 7) superiores a 50\% como um limite de aprovação dos produtos. Para todas as preparações desenvolvidas no presente estudo com inclusão do azeite de dendê, os valores ultrapassaram o limite de referência, como mostra a Tabela 3 .
Tabela 3. Percentual de provadores que atribuíram notas entre 5 e 7 pela escala hedônica de sete pontos para o atributo aceitação global, para os produtos originais e os desenvolvidos com azeite de dendê.

\begin{tabular}{|c|c|c|c|}
\hline \multirow[b]{2}{*}{$\begin{array}{c}\text { Amostra } \\
\text { (com dendê) }\end{array}$} & \multirow[b]{2}{*}{$\mathrm{n}$} & \multicolumn{2}{|c|}{$\%$ provadores } \\
\hline & & $\begin{array}{l}\text { Produto } \\
\text { original }\end{array}$ & $\begin{array}{c}\text { Produto } \\
\text { com azeite } \\
\text { de dendê }\end{array}$ \\
\hline Sequilho Salgado & 60 & $57,0^{\mathrm{a}}$ & $55,0^{a}$ \\
\hline Sequilho Doce & 62 & $66,1^{a}$ & $62,9^{a}$ \\
\hline Biscoito de Polvilho & 50 & $86,0^{\mathrm{a}}$ & $74,0^{\mathrm{a}}$ \\
\hline
\end{tabular}

\section{DISCUSSÃO}

Para verificar a viabilidade de uso do dendê como fonte de vitamina $A$, foram escolhidas preparações assadas, visto ser este tipo de produto o que atinge as mais altas temperaturas dentre as técnicas de preparação de alimentos. Como mostra a Tabela 1, a proporção de retenção de carotenoides ativos foi bem elevada, o que era esperado considerando ser a vitamina A relativamente estável ao calor ${ }^{[14]}$. Além disso, a elevada retenção do retinol nestes produtos foi determinada pela combinação do dendê com outros ingredientes, como farinha, açúcar e ovos, evitando sua exposição direta ao calor ${ }^{[15]}$.

Com relação à fortificação, a Portaria 31, da Agência Nacional de Vigilância Sanitária (ANVISA) ${ }^{[16]}$ estabelece que os alimentos que fornecem em $100 \mathrm{~g}$ de produto sólido pronto para o consumo no mínimo 15\% da Ingestão Diária Recomendada (IDR), podem ter o claim fonte, enquanto que para se ter o claim alto teor ou rico, é necessário que $100 \mathrm{~g}$ de produto sólido 
pronto para o consumo forneça, no mínimo, $30 \%$ da IDR. Diante dos resultados apresentados na Tabela 2, aos três produtos desenvolvidos poder-se-á atribuir o claim "alto teor/rico em vitamina A". Quanto à porção de $30 \mathrm{~g}$, a quantidade de vitamina A retida nas preparações desenvolvidas atenderá cerca de 15\% da IDR, ou seja, uma porção de $60 \mathrm{~g}$, ou o consumo de 2 porções diárias de $30 \mathrm{~g}$, darão às preparações o status de alimento enriquecido.

Obviamente, para terem valor, os produtos "fortificados" com vitamina A veiculada pelo azeite de dendê devem ter aceitação. Como mostra a Tabela 3 , os produtos desenvolvidos foram bem aceitos e não apresentaram diferenças estatisticamente significantes aos produtos controle (formulação original). Acrescenta-se, ainda, que o fato dos biscoitos não serem aceitos por todos os provadores não se deveu necessariamente à presença do azeite. $\mathrm{O}$ produto original, sem o azeite de dendê, também não foi apreciado pela totalidade dos comensais.

Assim, os resultados encontrados permitem dizer que a adição de azeite de dendê proporciona aos produtos, mesmo se submetidos a processamento térmico, que se tornem fontes de vitamina $\mathrm{A}$ e com uma boa aceitação sensorial.

Como a ingestão dietética deficiente é o fator determinante da DVA, o aumento do consumo de vitamina A se torna uma importante forma para combater sua deficiência e uma aliada no cumprimento do compromisso brasileiro em eliminar sua deficiência e suas consequências deletérias à saúde humana.

Em 1992, o Brasil assumiu um compromisso junto às Nações Unidas de propiciar para a população nacional - em relação à vitamina $\mathrm{A}$ - uma condição aceitável em níveis epidemiológicos definidos pela Organização Mundial de Saúde ${ }^{[17]}$. A data proposta para cumprimento do compromisso foi $2000^{[5-6]}$, posteriormente postergada para 2015 (Metas de Desenvolvimento do Milênio).

Conscientes da importância da hipovitaminose A para o desenvolvimento de crianças, em 1983, ou seja, 10 anos antes do compromisso firmado pelo Brasil para o controle da endemia, o Ministério da Saúde implantou um programa de suplementação de vitamina A destinado a crianças com 6 a 59 meses de idade, das áreas onde a desnutrição já havia sido caracterizada como endêmica:
Nordeste e Vale do Jequitinhonha na região Sudeste. $\mathrm{O}$ suplemento de vitamina A seria veiculado durante as campanhas de vacinação através de cápsulas contendo 100.000 UI (Unidades Internacionais) para crianças de 6 a 11 meses e de 200.000 UI para as de 12 a 59 meses de idade ${ }^{[18]}$.

A implementação do programa deixou a desejar. Como relatou Diniz ${ }^{[19]}$, em reunião realizada no Ministério de Saúde, em 1993, a distribuição do suplemento foi feita de forma regular exclusivamente no Estado da Paraíba. Nos outros Estados, o suplemento foi fornecido de forma irregular e, somente no Ceará e na Bahia, ele teve continuidade por mais de um ano.

Certamente a suplementação profilática na intervenção do controle de um nutriente é a forma mais econômica e simples de reverter uma situação de deficiência. No entanto, em relação à vitamina $\mathrm{A}$, a experiência nordestina de oferta de doses maciças do nutriente esbarra nas dificuldades inerentes à administração do programa que, além de tudo, é limitado a crianças.

Por sua vez, o quadro apresentado pela PNDS exige respostas imediatas de forma a minimizar e reverter o estágio da DVA, que já ultrapassou aquele da deficiência alimentar, tido como um indicador precoce da deficiência. A ingestão de alimentos em quantidade suficiente para atender à demanda biológica vem se distanciando da prática alimentar nacional e, também, da expectativa de consegui-la. Como mostra a Pesquisa de Orçamentos Familiares (POF) ${ }^{[20]}$, alimentos fontes de vitamina A préformada ou de equivalentes de retinol, estão sendo consumidos em quantidades muito reduzidas em relação à recomendação. Comparando as quantidades recomendadas de alimentos fontes de vitamina A com aquelas referidas na POF, verifica-se que a "segurança alimentar", no que se refere à vitamina $\mathrm{A}$, está muito distante de ser atingida: ao invés das 3 porções diárias de alimentos lácteos recomendados, o consumo no Brasil é de 0,8 porção; verduras/legumes e frutas, igualmente, ao invés das 3 porções recomendadas tem referida 0,5 porção. Entre as famílias de renda inferior a 2 salários mínimos, estes valores são, respectivamente: 0,4 para lácteos; 0,3 para verduras/ legumes e, para frutas, 0,2 de porção.

Mesmo que seja rotineiro o consumo de 
fontes de carotenoides com atividade de retinol, se a quantidade ingerida for irrisória, não atenderá sequer à demanda diária e, portanto, não estará apta a formar reservas do nutriente. Neste sentido, Fisberg e Barros ${ }^{[21]}$ chamam a atenção para a aquisição per capita de frutas e hortaliças na região Norte, de $38 \mathrm{~kg} /$ ano ou cerca de $100 \mathrm{~g} /$ dia, o que representa $25 \%$ da recomendação mínima diária correspondente a $400 \mathrm{~g}$. Uma avaliação feita pelo Ministério da Saúde em 14 mil crianças entre dois e cinco anos atendidas pelo Sistema Único de Saúde (SUS), em 2008, aponta que apenas $25,2 \%$ delas consomem frutas entre cinco e sete vezes por semana. A situação também é grave entre crianças de cinco a dez anos: de um total de 15 mil crianças entrevistadas, apenas 38,3\% informaram consumir frutas diariamente ${ }^{[22]}$.

Os produtos desenvolvidos neste estudo mostram algumas possibilidades, dentre diversas, que o dendê oferece no controle da DVA. O seu uso permite fornecer um significativo porcentual da IDR do nutriente sem maiores modificações no custo do produto/refeição, em função da pequena quantidade a ser acrescentada ao produto final.

A técnica de preparação escolhida não necessariamente precisa ser mantida. Alimentos de ingestão diária, como o feijão, podem ser o veículo para a fonte de retinol. Igualmente, o azeite pode ser adicionado ao alimento já preparado no momento da ingestão, e assim manter o seu potencial de atividade vitamínica sem sofrer qualquer perda.

Há que ressaltar, no que se refere à deficiência de vitamina $A$, que programas de suplementação profilática específicos para crianças pouco representariam para a população de risco que é bem mais abrangente. Vannucchi et al. destacam no relatório do PNDS ${ }^{[5]}$ que as prevalências de crianças e mulheres com níveis baixos e marginais de vitamina A estão muito elevadas e que "estratégias no âmbito da política nacional de alimentação e nutrição devem ser aperfeiçoadas para melhorar as condições de nutrição e saúde das crianças e mulheres em idade fértil, repercutindo no desenvolvimento do país como um todo". Se as consequências desta desnutrição são mais severas e visíveis entre crianças em idade préescolar, a hipovitaminose também está presente em proporções significativas entre indivíduos de outros grupos populacionais.
Diante desta situação, a adição do azeite de dendê, importante fonte de vitamina A a uma preparação, quer em domicílio, em instituições (onde alimentos são oferecidos como parte do atendimento), quer em produtos industrializados, poderia estar suprindo a população carente com o nutriente necessário para a sua saúde.

\section{CONCLUSÃO}

Ao se escolher o azeite de dendê como o composto veiculador da vitamina A no presente estudo, levou-se em consideração o fato deste ser um produto nacional, fonte relevante do nutriente em pauta e ainda, por poder ser utilizado em quantidades mínimas em preparações dietéticas sem alterar significativamente o sabor do produto, além de contribuir na melhora do seu aspecto visual.

Os resultados encontrados permitem dizer que o azeite de dendê se manteve como fonte de vitamina A quando utilizado como ingrediente de preparações que passam por um processamento térmico, mostrando ser uma excelente fonte complementar deste nutriente.

Além disso, as preparações propostas neste estudo são simples, fáceis de monitorar, acessíveis, com boa retenção de vitamina A e boa aceitação sensorial podendo, portanto, serem implantadas em centros, institutos, creches e outros órgãos, com vistas a estender os benefícios que os alimentos enriquecidos oferecem a toda a população, principalmente aos grupos de baixa renda/infantil, os mais vulneráveis à hipovitaminose $\mathrm{A}$.

Para atingir a população de forma mais abrangente, seria importante incorporar e medir a estabilidade da vitamina A do dendê em produções industriais, necessitando, para tanto, estudos complementares.

\section{REFERÊNCIAS}

1. Brasil ALD, Morais MG. Hipovitaminose A: prevalência, diagnóstico e tratamento. In: Cardoso AL, Lopes LA, Taddei JA de AC. Tópicos atuais em nutrição pediátrica. São Paulo: Sociedade de Pediatria de São Paulo; 2004. p. 45-57.

2. Brasil. Ministério da Saúde. Unicef. Cadernos de Atenção Básica: Carências de micronutrientes. Brasília: 
Ministério da Saúde, 2007. 60 p. (Série A. Normas e Manuais Técnicos).

3. Sarni RS, Kochi C, Ramalho RA, Schoeps DO, Sato K, Mattoso LCQ et al. Vitamin A: blood level and dietetics intake in stunted children and adolescents without hormonal disease. Rev Assoc Med Bras. 2002;48(1):48-53.

4. Geraldo RRC, Paiva SAR, Pitas AMCS, Godoy I, Campana AO. Distribuição da hipovitaminose A no Brasil nas últimas quatro décadas: ingestão alimentar, sinais clínicos e dados bioquímicos. Rev Nutr. 2003;16(4):443-60.

5. Brasil. Ministério da Saúde. Pesquisa Nacional de Demografia e Saúde da Criança e da Mulher - PNDS 2006: dimensões do processo reprodutivo e da saúde da criança/ Ministério da Saúde, Centro Brasileiro de Análise e Planejamento. Brasília: Ministério da Saúde, 2009. 300 p.: il. (Série G. Estatística e Informação em Saúde).

6. Batista Filho M, Rissin A. Deficiências nutricionais: ações específicas do setor de saúde para o seu controle. Cad Saude Publ. 1993;9(2):130-5.

7. Azevedo MMS, Cabral PC, Diniz AS, Fisberg M, Fisberg RM, Arruda IKG. Deficiência de vitamina A em pré-escolares da cidade do Recife, Nordeste do Brasil. ALAN. 2010;60(1):36-41.

8. Ebong PE, Owu DU, Isong EU. Influence of palm oil (Elaesis guineensis) on health. Plant Foods Hum Nutr. 1999;53(3):209-22.

9. Nagendran B, Unnithan UR, Choo YM, Sundram K. Characteristics of red palm oil, $\alpha$-carotene- and vitamin E-rich refined oil for food uses. Food Nutr Bull. 2000;21(2):189-93.

10. Sambanthamurthi R, Sundram K, Tan Y-A. Chemistry and biochemistry of palm oil. Prog Lipid Res. 2000;39(6):507-58.

11. Carvalho PRN, Collins CA, Rodriguez-Amaya DB. Comparison of provitamin A determination by normalphase gravity-flow chromatography and reversed phase high performance liquid chromatography. Chromatographia. 1992;33(3-4):133-37.

12. Dutcosky SD. Análise sensorial de alimentos. $3^{\mathrm{a}}$ ed. Curitiba: Champagnat; 2011.
13. Brasil. Ministério da Saúde. Agência Nacional de Vigilância Sanitária. Resolução RDC no 269, de 22 de setembro de 2005. Regulamento técnico sobre Ingestão Diária Recomendada (IDR) para proteína, vitaminas e minerais. Diário Oficial da União, Brasília, 23 set. 2005. Seção 1, p. 372.

14. Roncada MJ. Vitaminas lipossolúveis. In: Dutra-deOliveira JE, Marchini JS. Ciências nutricionais. São Paulo: Sarvier, 1998. p. 167-77.

15. Manorama R, Rukmini C. Effect of processing on $\beta$-carotene retention in crude palm oil and its products. Food Chem. 1991;42(3):253-64.

16. Brasil. Ministério da Saúde. Agência Nacional de Vigilância Sanitária. Portaria no 31, de 13 de janeiro de 1998. Regulamento técnico referente a alimentos adicionados de nutrientes essenciais. Diário Oficial da União, Brasília, 16 jan. 1998. Seção 1, p. 4.

17. World Health Organization. Indicators for assessing vitamin A deficiency and their application for monitoring and evaluating interventions programs. Micronutrients Series. Geneva: WHO/UNICEF; 1996.

18. Martins MC, Oliveira YP de, Coitinho DC, Santos LMP. Panorama das ações de controle da deficiência de vitamina A no Brasil. Rev Nutr. 2007;20(1):5-18.

19. Diniz AS. Combate à deficiência de vitamina A: linhas de ação e perspectivas. Rev Bras Saude Mater Infant. 2001;1(1):31-6.

20. Instituto Brasileiro de Geografia e Estatística [homepage]. Pesquisa de Orçamentos Familiares POF 2002-2003. Análise da disponibilidade domiciliar de alimentos e do estado nutricional no Brasil [acesso em 11 jun 2011]. Disponível em: http://www.ibge. gov.br/home/estatistica/populacao/condicaodevida/ pof $/ 2003$ medidas/pof 2003 medidas.pdf

21. Fisberg M, Barros MJL. Introdução. In: Fisberg M, Barros MJL. O papel dos nutrientes no crescimento e desenvolvimento infantil. São Paulo: Sarvier; 2008. p. $1-10$.

22. Brasil. Portal da Saúde [homepage]. MS incentiva a criação de políticas de alimentação saudável. Brasília: Ministério da Saúde [acesso em 27 set 2010]. Disponível em: http:// portal.saude.gov.br/portal/saude/default.cfm 